(c) 2018 Universidad Nacional Autónoma de México, Facultad de Estudios Superiores Zaragoza.

Este es un artículo Open Access bajo la licencia CC BY-NC-ND (http://creativecommons.org/licenses/by-nc-nd/4.0/).

TIP Revista Especializada en Ciencias Químico-Biológicas, 21(Supl. 1): 101-111, 2018.

DOI: 10.22201/fesz.23958723e.2018.0.147

\title{
El desarrollo de la biología molecular en América latina: Los casos de Argentina, Brasil, Cuba y México
}

\author{
${ }^{19}$ Alfonso Vilchis-Peluyera, aLuisa Alba-Lois, \\ ${ }^{b}$ Angeles Cancino-Rodezno aViviana Escobar-Sánchez, \\ aClaudia Segal-Kischinevzky y ${ }^{2 a}$ Víctor Valdés-López \\ aLaboratorio de Biología Molecular y Genómica. Departamento de Biología \\ Celular, Facultad de Ciencias, Universidad Nacional Autónoma de México, \\ Ciudad Universitaria, Deleg. Coyoacán, Ciudad de México, 04510, México. \\ '⿳亠口冋口十 Taller de Biología Molecular de la Célula I y II. Departamento de Biología \\ Celular, Facultad de Ciencias, Universidad Nacional Autónoma de México. \\ Correo electrónico: 1ajvp@ciencias.unam.mx, ${ }^{2}$ vvaldes@unam.mx
}

\section{RESUMEN}

Existen pocos estudios sobre el desarrollo de la biología molecular en América Latina. El propósito de este trabajo es presentar una narrativa histórica de la expansión de la biología molecular en aquellos países donde ha tenido un mayor desarrollo: Argentina, Brasil, Cuba y México. En estos países, la biología molecular inicialmente derivó de áreas como la bioquímica y la fisiología celular, aunque más tarde, con el advenimiento de la genética molecular y la ingeniería genética, se consolidó como una disciplina por sí misma. Los principales campos de investigación en biología molecular en América Latina han sido los siguientes: genética microbiana, biología molecular de la célula, vacunas recombinantes, ingeniería genética de plantas, manipulación molecular de vías metabólicas, generación de anticuerpos modificados contra venenos de serpientes y alacranes (faboterápicos) y en la última década, el establecimiento de proyectos de secuenciación de genomas de bacterias, plantas y parásitos.

Palabras Clave: desarrollo de la ciencia en América Latina, biología molecular, genómica, biología molecular y educación en América Latina.

The Development of Molecular Biology in Latin America: The Cases of Argentina, Brazil, Cuba and Mexico

\begin{abstract}
There are few studies on how molecular biology was developed in Latin America. The purpose of this research is to present a historical narrative about the expansion of molecular biology in countries where it has had a greater development: Argentina, Brazil, Cuba and Mexico. In these countries, molecular biology initially derived from areas such as biochemistry and cell physiology, but later, with the development of molecular genetics and genetic engineering, it became a consolidated discipline. The main fields of research in molecular biology in Latin America have been: microbial genetics, molecular cell biology, recombinant vaccines, genetic engineering of plants, molecular manipulation of metabolic pathways, generation of modified antibodies against snakes and scorpions poisons (fabotherapics) and, in the last decade, the establishment of genome sequencing projects of bacteria, plants and parasites.
\end{abstract}

Key Words: science development in Latin America, molecular biology, genomics, molecular biology and education in Latin America.

Nota: Artículo recibido el 04 de diciembre de 2017 y aceptado el 07 de mayo de 2018. 
Deseamos dedicar este trabajo en memoria de nuestro amigo y excepcional persona, el Dr. Federico Sánchez.

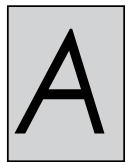

unque es un lugar común decir que la biología molecular nació con la publicación del modelo sobre la estructura del DNA por James Watson y Francis Crick en 1953, lo cierto es que la biología molecular ya había hecho su aparición desde la década de 1930 a 1940, con las investigaciones sobre estructuras de las proteínas, llevadas a cabo utilizando técnicas de cristalografía de rayos $\mathrm{X}$ por William Astbury y John D. Bernal en Gran Bretaña, los estudios sobre genética molecular de fagos y bacterias desarrollados por Salvador Luria y Max Delbrück a mediados de la década de 1940 a 1950 y con los experimentos bioquímicos de Oswald Avery y colaboradores, sobre la naturaleza química del principio transformante hacia 1944 (Hess, 1970; Judson, 1979). Desde luego que el modelo de Watson - Crick marcó el principio de una revolución en la biología, iniciando un programa de investigación nunca antes visto en las ciencias de la vida y sólo comparable con el de la física en las primeras décadas del siglo XX, con el desarrollo de la teoría de la relatividad y la mecánica cuántica. Es un hecho curioso -a nivel de historia de la ciencia y de la sociología de la ciencia-, que el primer premio Nobel otorgado sobre investigaciones en los ácidos nucleicos, después de la publicación del modelo de la estructura del DNA en 1953, no se le concediera a James Watson y Francis Crick sino a Severo Ochoa y Arthur Kornberg en 1959, por sus descubrimientos acerca de los mecanismos de la síntesis biológica de los ácidos ribonucleico y desoxirribonucleico; esto sucedió tres años antes del otorgamiento del premio Nobel a Watson, Crick y Maurice Wilkins.

A partir de la década de 1960, la biología molecular tuvo un gran desarrollo tanto en Europa -Francia y Gran Bretaña, principalmente-, como en los Estados Unidos, consolidándose como una disciplina independiente de la bioquímica y de la genética tradicionales. En América Latina, la biología molecular se desarrolló en torno a los laboratorios de bioquímica, pues esta última disciplina venía consolidándose en dos países americanos que contaban con una tradición, joven pero vigorosa, en investigación biomédica: Argentina y México.

Por su parte, en los casos de Brasil y Cuba, la Biología Molecular inició su desarrollo hacia 1980, vinculada a la aplicación de las técnicas de ingeniería genética y genómica para la resolución de problemas de tipo médico y agronómico (Ramírez et al., 2002; Rabinowicz, 2001).

\section{El caso de Argentina}

En Argentina las ciencias biomédicas tuvieron su despegue con la obra del Dr. Bernardo Houssay en fisiología, principalmente sobre los mecanismos hormonales que regulan el metabolismo de los carbohidratos; esta investigación llevó al Dr. Houssay a descubrir la participación de la glándula hipófisis en el desarrollo de la diabetes y su relación con los mecanismos de acción de la insulina (Nobel Lectures, 1964). El Dr. Houssay funda en 1923 la Sociedad Argentina de Biología, caja de resonancia de la mejor investigación biomédica no sólo de Argentina sino de la comunidad internacional a través de sus publicaciones, y en 1944 funda, junto con otros investigadores de primer nivel, el Instituto de Biología y Medicina Experimental, cuyo propósito sería el desarrollar investigación básica en biología y medicina. Es de subrayar que este Instituto se funda con fondos privados y fue intención del Dr. Houssay desvincularse de las subvenciones estatales con el fin de lograr una independencia académica y científica nunca antes vista en la ciencia argentina. Lo anterior se explica por el hecho de que la creación de este Instituto privado se dio en un contexto político muy adverso para el desarrollo de la ciencia en Argentina. El Instituto más tarde pasaría a formar parte de la Universidad de Buenos Aires (UBA) y es, hasta el momento, una de las instituciones científicas de mayor prestigio del país al "promover la investigación y la formación de recursos humanos en aspectos básicos en el campo de la oncología, endocrinología, reproducción, neurociencias y biotecnología orientados a ampliar el conocimiento de los principios fundamentales que rigen el funcionamiento de los seres vivos y desarrollar aplicaciones tecnológicas" (IBYME, 2016). Actualmente, y debido al perfil de la investigaciones realizadas y a los requerimientos presupuestales, el Instituto de Biología y Medicina Experimental se vincula al Consejo Nacional de Investigaciones Científicas y Tecnológicas de Argentina (CONICET), mismo que el propio Dr. Bernardo Houssay instituyó y presidió en Argentina, y que depende del Ministerio de Ciencia, Tecnología e Innovación Productiva de ese país; es de señalar el hecho de que el Instituto de Biología y Medicina Experimental refleja en su trayectoria las circunstancias, adversas unas veces y otras favorables, por las que ha transitado la ciencia en Argentina y en las cuales el propio Bernardo Houssay se vio involucrado (Cereijido, 2000). Por sus trabajos en endocrinología, en 1947 se le otorga el Premio Nobel de Fisiología o Medicina a Bernardo Houssay, junto con Carl y Gerti Cori, por sus trabajos sobre el metabolismo de la glucosa.

Otra de la figuras clave en el impulso a las ciencias biomédicas en Argentina, fue el Dr. Luis F. Leloir, discípulo de Bernardo Houssay y Premio Nobel de Química en 1970 por sus investigaciones sobre los azúcar-nucleótidos y su papel en la biosíntesis de carbohidratos. Una derivación de estos trabajos fue el descubrimiento de los pasos enzimáticos involucrados en la degradación de la galactosa hasta glucosa, hoy en día conocidos como vía de Leloir. Además de su importancia en ciencia básica, el aporte para la medicina clínica de esta investigación bioquímica lo representa la comprensión de las galactosemias, o patologías asociadas a la incapacidad de metabolizar la galactosa. De lo anterior, es evidente que la línea de investigación de Leloir y sus colaboradores se sitúa en la 
más pura tradición bioquímica. En 1947, Luis Leloir funda, con fondos privados, el Instituto de Investigaciones Bioquímicas de la Fundación Campomar, siguiendo el mismo esquema del Instituto fundado tres años antes por Houssay. Más tarde, el Instituto Campomar cambiaría de nombre a Fundación Instituto Leloir. Sus actividades principales se centran en investigaciones en bioquímica y biología celular y molecular. En la actualidad, la Fundación Leloir "está estrechamente relacionada con el CONICET, la UBA, la Universidad Nacional de Quilmes (UNQ) y la Universidad Nacional de San Martín (UNSAM)" (Leloir, 2016).

Continuando la tradición en fisiología, principalmente en endocrinología, aparece el nombre de Eduardo De Robertis, un médico que inició sus investigaciones sobre los mecanismos de secreción de la glándula tiroides, aclarando las vías de secreción de las hormonas tiroxina y triyodotironina a partir de la proteólisis de la molécula precursora tiroglobulina; esta investigación la llevó a cabo en el año de 1940 en la Universidad Johns Hopkins en Baltimore junto con Isidore Gersch (Barrios,1992). En 1942, De Robertis demostró que la secreción hipofisaria de la hormona gonadotrofina provoca, en sapos machos, la liberación de espermatozoides; este descubrimiento fue utilizado por un colega de De Robertis, Carlos Galli Mainini, para desarrollar la prueba de embarazo en seres humanos, también llamada reacción de Galli Mainini, consistente en la inyección de la orina de una mujer embarazada en el saco linfático dorsal de sapos machos, para estimular la secreción de espermatozoides (Enciclopedia de Ciencias, 2016).

Con el desarrollo de la microscopía electrónica en los Estados Unidos en la década de 1940 - 1950, el Dr. Eduardo De Robertis se interesó en la aplicación de esta técnica para el estudio de la estructura celular en células nerviosas, dando un giro sus investigaciones desde la endocrinología hasta la neurobiología y es así que, mediante el empleo de técnicas de microscopía electrónica, descubre, en 1953, la existencia de microtúbulos en células nerviosas y dos años después, trabajando entre la Universidad de Washington en Seattle con Henry Stanley Bennett y la Universidad de Montevideo, en Uruguay, descubre la presencia de vesículas en las regiones sinápticas en el tejido nervioso de la lombriz de tierra, demostrando la individualidad morfológica de las células neuronales, la cual había sido postulada por Santiago Ramón y Cajal en los inicios del siglo XX (Wells, 2005).

A su regreso a Argentina a mediados de los años 50, el Dr. De Robertis continuó sus investigaciones en neurobiología, las cuales se vieron obstaculizadas por las intervenciones militares en 1966 y una década después por el golpe militar, que dispersó a los científicos argentinos, enviando a muchos de ellos al exilio. Estos golpes militares afectaron primordialmente a los centros universitarios, lo cual dificultó en grado máximo el desarrollo de la investigación científica (Leloir, 2016).
Las líneas de investigación antes señaladas marcan el desarrollo de la etapa "clásica" de las ciencias biomédicas en Argentina, pudiéndose afirmar que las investigaciones en fisiología y bioquímica, a partir de 1920, fueron la plataforma científica para el desarrollo de las ciencias biomédicas en este país, que ambas disciplinas se desarrollaron al principio en contextos académicos y científicos privados y, por lo tanto, al margen de lineamientos gubernamentales u oficialistas, consolidándose grupos de investigación de gran prestigio, que, con el tiempo y pasadas las asonadas militares, fueron la base de instituciones científicas de primer nivel que ya contaron con un apoyo académico y presupuestario a nivel gubernamental. A este respecto es importante mencionar el caso de la trayectoria del Dr. César Milstein, doctorado en química por la Universidad de Buenos Aires, que realizó su tesis estudiando a la enzima aldehído deshidrogenasa hacia mediados de la década de 1950, bajo la dirección del profesor Andrés Manuel Stoppani, discípulo de Bernardo Houssay (Stoppani \& Milstein, 1957). En 1958 recibió una beca para estudiar en el Departamento de Bioquímica de la Universidad de Cambridge en Inglaterra, en donde trabajaba Frederick Sanger; allí realizó su segundo doctorado bajo la dirección de Malcom Dixon sobre enzimología (Neuberger, 2002). Después de retornar dos años a Argentina, Milstein regresó a la Universidad de Cambridge para trabajar con Frederick Sanger en química de proteínas, dando posteriormente un giro hacia la investigación con anticuerpos en asociación con Georges Köhler, con quien escribió, en 1975, su famoso artículo sobre la producción experimental de anticuerpos monoclonales a partir de la fusión de linfocitos B con células de mieloma, los llamados hibridomas (Neuberger, 2002). Por sus trabajos sobre inmunología aplicada, César Milstein recibió en 1984 el premio Nobel junto con Niels Jerne y Georges Köhler. Aunque Milstein nunca regresó a trabajar en Argentina, siempre mantuvo lazos con la comunidad científica de su país de origen.

La continuidad científica argentina en las áreas biomédicas pasó de las manos de Luis Leloir a las de Héctor Norberto Torres, discípulo suyo y de Houssay. El Dr. Héctor Torres fue el impulsor de la biología molecular y la ingeniería genética en Argentina a partir de 1980, formando grupos de investigación en áreas como la transducción de señales mediadas por el sistema de la adenil ciclasa y el AMPc, tomando como modelo al hongo Neurospora crassa, y posteriormente ampliando sus estudios hacia los mecanismos de conversión patogénica en Trypanosoma cruzi (protista causante de la enfermedad de Chagas) y los mecanismos moleculares del desarrollo del parásito, también involucrando transducción de señales mediante proteínas G. Todas estas investigaciones en la biología molecular de Trypanosoma cruzi han conducido a una mejor comprensión de los mecanismos de invasión del parásito $\mathrm{y}$, por lo tanto, a una búsqueda de blancos terapéuticos. El Dr. Héctor Torres fundó el Instituto de Ingeniería Genética y Biología Molecular de Argentina en 1983, el cual tuvo su origen en el Laboratorio de Regulación Metabólica de la "Fundación Campomar", ya 
mencionada, dirigido por él mismo (Birnbaumer, 2011). De esta manera se fue dando la transición entre los programas de investigación bioquímica y las nuevas áreas de la biología, a las cuales se incorporaron, a partir del año 2000, proyectos en genómica como el análisis genómico de la planta de girasol, la secuenciación del genoma de Brucella abortus -un patógeno del ganado vacuno-, hasta la incorporación de metodologías de secuenciación completa de genomas (WGS) aplicada en el año 2015 para la identificación de una nueva mutación genética ligada al autismo en tres hermanos que padecían este síndrome (Rabinowicz, 2001; Nemirovsky et al., 2015).

De esta manera, observamos la continuidad de la comunidad biomédica en Argentina y la importancia de generar núcleos de investigación sólidos, los cuales, al irse diversificando, van desarrollando nuevas áreas de trabajo y fundando o expandiendo nuevas disciplinas. Como veremos más adelante, el caso de México muestra semejanzas con este país del Cono Sur.

Respecto a la situación actual de la genómica en Argentina-como una de las derivaciones más importantes de la biología molecular-, en el año 2003 se funda el Centro Regional de Estudios Genómicos en la Universidad de la Plata, con apoyo del Instituto Max Planck de Alemania; en este Instituto, científicos de la Asociación de Universidades del Grupo Montevideo(AUGM) -albergando a 15 instituciones de Argentina, Brasil, Chile, Paraguay y Uruguaydesarrollan investigaciones sobre genomas de diferentes especies, incluyendo organismos de importancia médica. Es de destacar el hecho de que existe una red de laboratorios por todo el país dedicados a unir esfuerzos en la secuenciación parcial de genomas de organismos como Trypanosoma cruzi, Leishmania major y Schistosoma mansoni (Rabinowicz, 2001) y en el año 2011 se anunció el primer genoma, secuenciado en Argentina, de un organismo extremófilo presente en lagos volcánicos de los Andes, Sphingomonas sp. S17. Como resultado de este esfuerzo de secuenciación genómica, se identificaron diversos genes involucrados en la resistencia a elevados niveles de radiación ultravioleta, genes relacionados con el metabolismo del azufre, resistencia a antibióticos y adaptación a elevados porcentajes de salinidad y condiciones alcalinas. Lo anterior fue resultado de un esfuerzo conjunto de cinco laboratorios argentinos que trabajaron con financiamiento nacional en su totalidad (Farías et al,2011). Otro proyecto de secuenciación genómica fue llevado a cabo en la gammaproteobacteria Acinetobacter sp. Ver 3, aislada del Lago Verde en los Andes, a una altitud de 4,400 msm; este proyecto de secuenciación tuvo por objetivo identificar genes asociados con la resistencia a elevada exposición a la radiación ultravioleta; esta bacteria es considerada un organismo modelo en el estudio de la adaptación a condiciones de irradiación ultravioleta extrema (Kurth et al., 2015).

Con respecto al área biomédica, en Argentina se secuenció, en el año 2014, el genoma de la bacteria Acinetobacter baumannii, causante de diversas infecciones intrahospitalarias; este microorganismo patógeno presenta múltiple resistencia a antibióticos así como elementos genéticos vinculados con la transferencia horizontal, fenómeno asociado a la diseminación de genes de resistencia a fármacos en poblaciones bacterianas (Traglia et al, 2014).

En el año 2014, Argentina fue el primer país latinoamericano que se asoció con el Laboratorio Europeo de Biología Molecular (EMBL, por sus siglas en inglés). El EMBL fue ideado como un centro internacional de biología molecular por Leo Szilárd, Victor F. Weisskopf, James D. Watson y John Kendrew en 1962 y abierto en 1974 en Heidelberg, Alemania. Las principales áreas en las que Argentina participará en el EMBL serán la genómica y la bioinformática, desarrollando proyectos conjuntos con países europeos (EMBL, 2014).

\section{El CASO de Brasil}

Otro país sudamericano en el cual la genómica ha tenido un sustento institucional nacional de gran relevancia a partir de la década de 1990, es Brasil, donde en el año 2000 se secuenció por primera vez el genoma de la bacteria Xyllela fastidiosa, un microorganismo fitopatógeno causante de la pérdida del $30 \%$ de las cosechas de cítricos en Brasil, principal país exportador de concentrado de jugo de naranja a nivel mundial (Simpson et al., 2000). En este esfuerzo científico de secuenciación participaron treinta laboratorios brasileños, coordinados por una institución "virtual", ONSA (Organization for Nucleotide Sequencing and Analysis), es decir, una red de intercambio de información vía Internet entre diferentes laboratorios e investigadores que participaron en ese proyecto genómico. Este sistema de colaboración inter-institucional, al demostrar su eficacia, ha permitido que Brasil emprenda nuevos proyectos de secuenciación de microorganismos patógenos de importancia agrícola, como Leifsonia xyli, bacteria Gram positiva causante del raquitismo de la caña de azúcar y de Xanthomonas citri, una proteobacteria que infecta a una gran variedad de cítricos, provocando pérdidas millonarias en los cultivos (Rabinowicz, 2001; Monteiro-Vitorello et al., 2004). Esta estrategia de colaboración entre diferentes laboratorios vinculados a universidades e institutos de investigación ha resultado absolutamente satisfactoria para el caso de Brasil, puesto que ha permitido a) escoger el organismo adecuado para secuenciar su genoma, basándose en consideraciones de importancia práctica, b) la organización de redes de laboratorios, aunque sean pequeños, en lugar de establecer grandes laboratorios, haciendo uso de la infraestructura ya establecida, y c) integrar recursos financieros en un gran proyecto de secuenciación en vez de dispersarlos hacia proyectos genómicos parciales o a la caracterización de genes individuales en diversos microorganismos fitopatógenos (Rabinowicz, 2001).

Asimismo, recientemente Brasil ha incursionado en el área de la biotecnología médica, fundando el Centro Integrado de 
Prototipos, Biofármacos y Reactivos para Diagnóstico, así como el Instituto de Tecnología Inmunobiológica en Río de Janeiro, con el fin de desarrollar nuevas vacunas virales y bacterianas, la producción de alfa-eritropoyetina recombinante (epoetina) y el alfa-interferón recombinante humano; como parte de este esfuerzo, el gobierno brasileño espera producir anualmente, 50 millones de dosis de insulina humana recombinante para suplir, en parte, la importación de insulina, que alcanza la cifra de 170 millones de dosis al año (Bonalume-Neto, 2010).

Un problema de salud pública en muchos países de América Latina, Asia y África situados en regiones tropicales y subtropicales, lo representa la presencia del virus del dengue y su diseminación a través de los mosquitos vectores Aedes aegypti y Aedes albopictus, que lo transmiten a los seres humanos. La enfermedad del dengue puede mostrar dos patologías diferentes: el dengue febril (poco severo) y el dengue hemorrágico (muy severo, e incluso mortal). Este virus pertenece al género Flavivirus, con un genoma de RNA y muestra cuatro serotipos antigénicamente diferentes, denominados D1 -D4(Mantel et al., 2011). Respecto a los avances para el desarrollo de vacunas contra el virus del dengue, diversos centros de investigación biomédica en Brasil han unido esfuerzos con la compañía francesa SanofiPasteur, para la formulación de una vacuna tetravalente que incluye los cuatro serotipos del virus del dengue, D1 - D4; esta vacuna polivalente es de tipo recombinante y está formada por cuatro virus quiméricos generados por la recombinación del genoma del virus atenuado de la fiebre amarilla cepa $17 \mathrm{Y}$ con genes del virus del dengue. Los genes para la cubierta viral de la cepa 17 Y han sido reemplazados por genes estructurales de la cápside de cada uno de los cuatro serotipos del virus del dengue (Mantel et al., 2011). Esta vacuna fue probada entre los años 2013 - 2014 en cinco países de Latino América, donde el virus del dengue es endémico y se aplicó a un total de 20,859 niños. Se reportó una eficacia de la vacuna de 50.3\% para el serotipo 1, de $42.3 \%$ para el serotipo $2,74.0 \%$ para el serotipo 3 y de $77.7 \%$ para el serotipo 4 . La vacuna tetravalente está indicada tanto para el dengue febril como para el dengue hemorrágico (Villar et al., 2015). Otra vacuna contra el virus del dengue está en desarrollo avanzado y es fruto de la colaboración entre investigadores de diversos Institutos Nacionales de Salud de Estados Unidos y el Instituto Butantan en Sao Paulo, Brasil. Esta vacuna tetravalente consiste en una mezcla de los cuatro serotipos del virus del dengue atenuados por mutagénesis y expresan tanto proteínas estructurales de la envoltura viral como proteínas no estructurales; los ensayos clínicos de fase III en humanos comenzaron en febrero del año 2016 en Brasil y se espera que para el año 2018 la vacuna esté disponible en el mercado. El Instituto Butantan es el encargado de la elaboración de esta vacuna para los ensayos clínicos finales (Perkel, 2016).

\section{El CASO de Cuba}

Un país en el que las técnicas de biología molecular e ingeniería genética fueron rápidamente aplicadas al desarrollo de la biotecnología biomédica es Cuba, en donde, a partir de la década de 1980, la investigación básica y su vínculo con la elaboración de productos biofarmacéuticos han ido de la mano. Con la fundación del Centro de Ingeniería Genética y Biotecnología en 1986, predecesor del Centro de Investigaciones Biológicas en la ciudad de La Habana, este país ha venido generando diversos medicamentos novedosos, como el interferón alfa leucocitario semipurificado contra el dengue hemorrágico, que se aplicó por vez primera a nivel mundial contra esta enfermedad de manera exitosa en un brote de fiebre hemorrágica de dengue en 1981; no fue sino hasta 1987 cuando se produce por primera vez mediante técnicas de DNA recombinante con el nombre de HEBERONALFAR ${ }^{\circledR}$ (García, 2010). Además de su utilización contra el dengue hemorrágico, también se ha administrado en el tratamiento contra la hepatitis B y C y Herpes zoster y en la actualidad, se llevan a cabo investigaciones sobre sus propiedades antiproliferativas en el tratamiento de diversas neoplasias. Para el caso de la hepatitis B, Cuba desarrolló en 1991 su propia vacuna recombinante compuesta por el antígeno de superficie del virus en cuestión -aunque la vacuna ya había sido desarrollada en los Estados Unidos en 1986-. En 1992 comenzó su aplicación masiva en niños, y para 2010, todas las personas menores de 25 años ya habían sido vacunadas contra la hepatitis B en Cuba, empleando su propia vacuna desarrollada en el Centro de Ingeniería Genética y Biotecnología, HEBERVIOVAC HB $^{\circledR}$ (García, 2010).

Otro gran éxito de la biología molecular y la ingeniería genética en Cuba ha sido la clonación y expresión del gen del factor de crecimiento epidérmico humano mediante técnicas moleculares; este es un péptido de 53 residuos de aminoácidos que estimula la proliferación celular y los procesos de cicatrización, activando procesos mitogénicos en fibroblastos y células epiteliales. Este producto biológico ha sido utilizado principalmente en el tratamiento de la úlcera del pie diabético con gran eficacia terapéutica, tanto en Cuba como en otros 14 países, siendo este biofármaco el indicado a nivel clínico para el manejo de las complicaciones ulcerativas que se presentan en las extremidades inferiores de pacientes con este padecimiento. El producto ha sido registrado en 12 países con el nombre de Heberprot - $\mathrm{P}^{\circledR}$ (Fernández, Mena \& Santiesteban-Bonaechea, 2010) .

Aunque se podrían citar más ejemplos de biofármacos utilizados en el diagnóstico, prevención y tratamiento de diversos padecimientos, desarrollados principalmente por el Centro de Ingeniería Genética y Biotecnología de Cuba, basten los tres bioproductos antes mencionados para dar una idea del tipo de investigación realizada en este país en el área de la biología molecular y la ingeniería genética, integrando también las investigaciones de otros institutos científicos cubanos como el Instituto Finlay, el Centro de Inmunología Molecular, el Centro Nacional de Biopreparados, el Centro de Inmunoensayo y el Centro Nacional de Investigaciones Científicas. El desarrollo de biofármacos y otros productos generados por técnicas de 
DNA recombinante en Cuba es resultado de una modalidad de desarrollo biotecnológico denominado de ciclo cerrado, que involucra una primera etapa de investigación básica seguida del desarrollo y escalamiento a nivel laboratorio y planta piloto, continuándose con el proceso de producción industrial y una etapa final de comercialización, esto último mediante la obtención de patentes a nivel internacional y la exportación de los biofármacos a diversos países del mundo. Así, queda claro que, en el área biomédica, en Cuba no existe el dilema entre desarrollar una ciencia básica o desarrollar una ciencia aplicada, pues los proyectos de investigación tienen desde el principio un objetivo concreto y que es imposible el despliegue de una ciencia aplicada o biotecnológica si antes no se cuenta con la plataforma de conocimientos básicos o fundamentales necesarios para determinada aplicación. De esta manera se logra maximizar el esfuerzo científico y los requerimientos presupuestales de inversión.

\section{El CASO de México}

Por último, llegamos al caso de México, país en donde la biología molecular ha tenido un desarrollo, aunque no tan extensivo, sí muy intensivo, abarcando diversas disciplinas científicas como genética bacteriana, inmunología, parasitología, virología, microbiología, biología de membranas, neurobiología y biomedicina en general. Al igual que en el caso de Argentina, la biología molecular en México se desarrolla a partir de grupos de investigación que se formaron en el campo de la bioquímica y la biología celular y que, con el surgimiento y consolidación de diversas áreas de investigación en biología molecular a nivel mundial, estos pequeños grupos fueron asimilando los conceptos y metodologías en esta rama y definiendo líneas de investigación propias en las áreas arriba mencionadas.

Desde mediados de la década de 1930, comienza en la Facultad de Medicina de la UNAM, la introducción de cursos de Química Biológica, dependientes del Departamento de Fisiología y no es sino hasta 1956 que el nombre de Bioquímica como disciplina se incorpora formalmente en el Currículum de la carrera de Medicina. Entre los iniciadores de la bioquímica en México destacan los doctores José Laguna García y Jesús Guzmán García, cuya labor, tanto en docencia como en investigación, sentaron el desarrollo de amplios programas de investigación en diversas áreas de la bioquímica (Castañeda, 2002). Desde la década de 1940, México ya contaba con líneas de investigación de vanguardia en el área biomédica, principalmente en el campo de la fisiología del sistema nervioso con los trabajos de los doctores Arturo Rosenblueth Stearns, Efrén del Pozo Rangel y José Joaquín Izquierdo Raudón. También por esa época se consolidaba la investigación biomédica en el Instituto Nacional de Cardiología, con los trabajos experimentales del Dr. Ignacio Chávez Sánchez sobre cateterismo intracardíaco y angiocardiografía y los de electrocardiografía con el Dr. Demetrio Sodi Pallarés, los cuales colocaron a la cardiología desarrollada en México entre los primeros lugares a nivel mundial, reconocimiento que a la fecha se mantiene vigente. A partir de mediados de la década de 1950 y con el desarrollo de la bioquímica como una rama independiente, aunque relacionada, de la fisiología, se conforman grupos de investigación en la Facultad de Medicina y en la Facultad de Química de la UNAM. Un paso inicial muy importante para el desarrollo de la biología molecular en México fue la creación, en 1969, del Instituto de Investigaciones Biomédicas de la Universidad Nacional Autónoma de México (IIB-UNAM), descendiente directo del Instituto de Estudios Médicos y Biológicos, fundado en 1941 por el Dr. Ignacio González Guzmán, pionero de la hematología a nivel mundial. En el IIB se van a fundar, entre 1965 y 1975, los departamentos de Biología Molecular, Biología del Desarrollo y Biofísica y Biomatemáticas. En 1976, se establece el Departamento de Biotecnología, con dos divisiones: Biomedicina y Bioingeniería. Hacia 1981, la división de Biomedicina da origen al Departamento de Inmunología. Actualmente, la distribución de laboratorios se enmarca en cuatro áreas de investigación: Biología Celular y Fisiología, Biología Molecular y Biotecnología, Inmunología, Medicina Genómica y Toxicología Ambiental (Memoria UNAM, 2002).

Otra institución que desde su fundación en 1961 ha generado una labor de investigación biomédica de alto nivel, es el Centro de Investigación y Estudios Avanzados del Instituto Politécnico Nacional (CINVESTAV). Este Centro fue creado por iniciativa del Dr. Arturo Rosenblueth y, a partir de 1975, se establece el Departamento de Genética y Biología Molecular, que desarrolla investigaciones en genética molecular de bacterias, parasitología molecular, virología, regulación de la expresión génica en procariontes y eucariontes, entre otros.

Personajes importantes que impulsaron el establecimiento de la biología molecular en México fueron Guillermo Soberón Acevedo, como primer Director del IIB-UNAM apoyando la formación de grupos de investigación en esta área, Raúl Ondarza Vidaurreta (enzimología de parásitos), Jaime Mora Celis (metabolismo nitrogenado en Neurospora crassa), Jaime Martuscelli Quintana (genética molecular), Rafael Palacios de la Lama (parasitología molecular), Sergio Sánchez Esquivel (metabolismo microbiano), Carmen Gómez Eichelmann (mecanismos transcripcionales en E. coli), Beatriz Gómez García (virología molecular) y Lourival Possani Postay (toxinas) en la UNAM, entre otros, mientras que en CINVESTAV los pioneros fueron Fernando Bastarrachea Avilés (genética molecular bacteriana), Gabriel Guarneros Peña (regulación génica en el fago lambda, mecanismos de traducción en procariontes, minigenes en E. coli), Carlos Fernández Tomás (genética viral) y Jacobo Kuperztoch Portnoy (mecanismos de resistencia a antibióticos en bacterias patógenas) (Calva, 2009). Fue un investigador del Instituto de Investigaciones Biomédicas de la UNAM, el Dr. Francisco Bolívar Zapata, quien, a mediados de la década de 1970 y durante una estancia postdoctoral en el laboratorio del Dr. Herbert Boyer en la 
Universidad de California en San Francisco, realiza una de las aportaciones más importantes a la naciente ingeniería genética: la construcción de vectores de clonación y expresión tanto de DNA homólogo -de la propia Escherichia coli-, como de DNA heterólogo -proveniente de diversos orígenes-. Estos vectores de clonación multipropósito, conteniendo diversas secuencias como blanco de endonucleasas de restricción para sitios de clonación y marcadores de resistencia a antibióticos como elementos de selección de bacterias recombinantes, fueron el punto de partida para la posterior clonación y expresión de los genes sintéticos de la insulina y de la somatostatina humanas (hormona de crecimiento), primeros biofármacos -hormonas proteicas, en este caso-generados mediante ingeniería genética; lo anterior fue logrado entre 1977 y 1979 por el equipo de Herbert Boyer y Francisco Bolívar (Soberón, 1997). El prototipo de estos vectores construidos por Francisco Bolívar, el plásmido pBR322, fue el punto de partida para el desarrollo de diversos vectores de clonación y expresión, elementos clave de la moderna Biotecnología del DNA recombinante.

A su regreso a México, Francisco Bolívar integra un grupo de trabajo en el IIB-UNAM, que posteriormente será el núcleo del Centro de Investigación sobre Ingeniería Genética y Biotecnología, fundado en 1982 con el Dr. Bolívar como su primer director y más tarde (1992) convertido en el actual Instituto de Biotecnología (IBT-UNAM). En este Instituto se han consolidado diferentes grupos y líneas de investigación, realizando una labor científica y de docencia de un alto nivel; entre las áreas de investigación podemos mencionar las siguientes: biología molecular, celulary bioquímica de parásitos, biotecnología molecular y biotecnología de plantas, estructura, función y manipulación de péptidos y proteínas, genética del desarrollo y fisiología molecular, ingeniería de vías metabólicas, neurobiología celular y molecular y el área de bioinformática, entre otras (Instituto de Biotecnología, 2017). Una de las líneas de investigación en las que el grupo de trabajo del Dr. Francisco Bolívar ha tenido un fuerte impacto en la moderna microbiología biotecnológica es la ingeniería de vías metabólicas, la cual tiene como objetivo "la modificación orientada de las vías metabólicas originales de un microorganismo, para transformarlo en otro capaz de manejar de manera diferente los esqueletos de carbono y sus fuentes de energía, para poder así sobreproducir moléculas biológicas de interés" (Soberón, 1997). Un trabajo pionero en este campo fue publicado por el grupo de Francisco Bolívar en 1996, en el cual se describe la modificación experimental de la vía metabólica del fosfoenolpiruvato (PEP) y del sistema de fosfotransferasa de glucosa (PTS), para la sobreproducción de compuestos aromáticos en E. coli (Floras, Xiao, Berry, Bolivar \& Valle, 1996).

En 1980 se crea, también en la UNAM, el Centro de Investigación sobre Fijación del Nitrógeno, incorporando grupos de investigación en biología molecular de plantas, ecología molecular e ingeniería de vías metabólicas; en este Centro se utilizó como organismo modelo a la bacteria simbionte del frijol, Rhizobium etli, enfocándose la principal plataforma de investigación a conocer en profundidad la biología molecular de esta bacteria simbionte fijadora de nitrógeno. Entre los investigadores de este Centro que comenzaron el estudio de la biología molecular de la interacción simbionte-planta, destacan los doctores Federico Sánchez Rodríguez, Carmen Quinto Hernández, Miguel Lara Flores, Guillermo Dávila Ramos y David Romero Camarena, mientras que el desarrollo de las plataformas bioinformáticas se llevó a cabo bajo la dirección del Dr. Julio Collado Vides (Calva, 2009).

Debido a la incorporación de nuevas metodologías de análisis genómico para el estudio de este microorganismo, como la secuenciación de DNA, genómica funcional -entendida como el análisis de la expresión de genes o transcriptómica- y el análisis de las proteínas sintetizadas o proteómica, así como el desarrollo de herramientas bioinformáticas, en el año 2004, el Centro de Investigación sobre Fijación del Nitrógeno se transforma en el Centro de Ciencias Genómicas, el cual actualmente desarrolla investigaciones en áreas como genómica computacional, genómica evolutiva, genómica funcional de procariontes y eucariontes, ecología genómica, dinámica genómica e ingeniería genómica (Centro de Ciencias Genómicas, 2012). Uno de los primeros logros de este Centro fue la secuenciación, pese a fuertes limitaciones tanto en cómputo como en infraestructura, del genoma del plásmido simbiótico de la cepa tipo de Rhizobium etli en el año 2003 y la publicación de la secuencia completa del genoma de este microorganismo de gran importancia agronómica, en el año 2006 (González et al., 2006).

También en la UNAM se consolida como núcleo de investigación en biología molecular y neurofisiología el Instituto de Fisiología Celular (IFC-UNAM), el cual tuvo como antecesor el Departamento de Biología Experimental del Instituto de Biología y la Facultad de Medicina. En su inicio, confluyen las áreas de investigación centradas en el metabolismo celular, desarrolladas principalmente en la Facultad de Medicina y los programas de investigación en neurobiología, llevados a cabo en el Instituto de Biología (para una visión histórica del desarrollo del Instituto de Fisiología Celular de la UNAM, véase: Tapia, R. Breve historia del origen del Instituto de Fisiología Celular, 2016). Actualmente, el IFC integra amplios programas de investigación como Neurociencias, Bioquímica y Biología Estructural, Genética Molecular y Biología Celular y del Desarrollo, en áreas tan diversas como metabolismo nitrogenado en Saccharomyces cerevisiae y estructura de la cromatina, desarrolladas en el laboratorio de la Dra. Alicia González Manjarrez, estudios estructurales de enzimas, llevados a cabo por el Dr. Armando Gómez Puyou, regulación de la expresión génica durante el desarrollo y su relación con la estructura de la cromatina, área de investigación del Dr. Félix Recillas Targa, modulación del metabolismo intermedio hepático por adenosina en el laboratorio de la Dra. Victoria Chagoya Sánchez, estudios 
de los mecanismos de transporte de iones en Saccharomyces cerevisiae y adaptación a condiciones salinas en Debaryomyces hansenii (levadura marina), llevados a cabo por el grupo de investigación del Dr. Antonio Peña Díaz, entre otros muchos proyectos (Instituto de Fisiología Celular, 2017).

Otra aportación de primera importancia en el campo de la biología molecular y la ingeniería genética, la representa el trabajo del Dr. Luis Herrera Estrella, científico mexicano formado en la Escuela Nacional de Ciencias Biológicas del Instituto Politécnico Nacional y el CINVESTAV, quien realizó su investigación de doctorado en la Universidad de Gante, Bélgica, sobre los mecanismos de transformación genética en vegetales y generó, en 1983, la primera planta transgénica, incorporando genes bacterianos al genoma de la planta de tabaco Nicotiana tabacum. El Dr. Luis Herrera y su grupo emplearon para estos eventos de transformación genética a la bacteria Agrobacterium tumefaciens y su plásmido Ti, al cual le incorporaron las señales transcripcionales del gen de la nopalina sintetasa y un marcador de resistencia a cloranfenicol, el gen de la cloranfenicol acetiltransferasa; tanto el promotor de la nopalina sintetasa como el gen de resistencia eran de origen bacteriano; esta fue la primera demostración experimental del potencial de las técnicas de DNA recombinante para la modificación de las características genéticas de una planta (Herrera-Estrella, Depicker, Van Montagu \& Schell,1983; De Block, Herrera-Estrella, Van Montagu, Schell \& Zambryski, 1984). Este experimento marcó el nacimiento de la moderna biotecnología vegetal y el punto de partida de los vegetales transgénicos.

Como en el caso del Dr. Francisco Bolívar, Luis Herrera también regresó a México, pero a colaborar en el CINVESTAV-Unidad Irapuato, creando en 1986 el Departamento de Ingeniería Genética, con énfasis en la biología molecular de plantas. Desde el año 2001 al 2005 fue director del CINVESTAV-Unidad Irapuato y en el mismo año de 2005 funda el Laboratorio Nacional de Genómica para la Biodiversidad - LANGEBIO -, cuyo propósito es el de llevar a cabo investigación de frontera en el área de la genómica y, al mismo tiempo, generar conocimiento genético sobre la biodiversidad de México, que pudiera conducir a un uso racional y sustentable de sus recursos biológicos. Uno de los logros más recientes de este laboratorio fue la secuenciación del genoma de una variedad de maíz, el llamado "maíz palomero", cuya comparación con el genoma del maíz comercial, tipo B73, mostró regiones genéticas asociadas a los procesos de domesticación mediante selección artificial así como los componentes genéticos asociados a características fenotípicas apropiadas para su siembra y consumo (VielleCalzada et al., 2009; Schnable et al., 2009).

Por último, es importante señalar la fundación del Instituto Nacional de Medicina Genómica (INMEGEN) en el año 2004, que es una entidad de investigación biomédica centrada en estudios sobre la estructura genómica de subpoblaciones mexicanas y grupos étnicos y en sus características epidemiológicas; lo anterior tiene la finalidad de obtener información biomédica sobre los perfiles genómicos de poblaciones particulares y su relación con la incidencia de diversas patologías. Se espera que, como resultado de diversos programas de investigación sobre genómica poblacional, el INMEGEN pueda desarrollar métodos de diagnóstico clínico basados en información genómica y aplicar tratamientos de farmacogenómica individualizados.

Los resultados del primer estudio sobre la diversidad de los perfiles genómicos de subpoblaciones mexicanas como base para estudios de asociación con enfermedades complejas (poligénicas y multifactoriales) se publicaron en el año 2009 (Silva-Zolezzi et al., 2009), representando uno de los estudios más amplios de genotipificación a nivel genómico llevados a cabo en América Latina. Este es un ejemplo claro de la aplicación de la genómica a las ciencias de la salud en México.

Los casos anteriores son una muestra del desarrollo de programas de investigación en biología molecular, genómica y biotecnología en México, aunque otra parte muy importante en este desarrollo lo han representado los programas de enseñanza a nivel de licenciatura y posgrado, en los cuales se han incorporado materias como biología molecular, genómica, bioinformática y biotecnología, con relevancia en los planes de estudio de carreras como Biología, Química e Investigación Biomédica Básica, impartidas en instituciones como la Facultad de Ciencias, la Facultad de Química y el IIB de la UNAM, incluso el Centro de Ciencias Genómicas de la UNAM imparte la licenciatura en Ciencias Genómicas. De esta manera la formación de científicos en áreas como biología molecular, genómica, bioinformática y biotecnología ha tenido un auge muy importante en instituciones de educación superior como universidades y centros de investigación (Quintero, 2007).

\section{Conclusiones}

En esta breve revisión se ha podido ver cómo la biología molecular ha tenido un desarrollo desigual en América Latina, aunque también se observa una tendencia general, al igual que en otras partes del mundo, hacia una biología molecular más integrativa, transitando desde el estudio de los genes individuales y su expresión, hasta el desarrollo de la genómica, es decir, el análisis de la totalidad del DNA contenido en las células de un organismo, los mecanismos de regulación de la expresión génica (transcriptoma) y, a un nivel de integración más elevado, las redes de interacción entre proteínas (proteoma) que conforman las vías metabólicas (metaboloma), así como las cascadas de señalización al interior y al exterior de las células. Las experiencias de Brasil y México confirman esta tendencia. En resumen, se puede señalar que el desarrollo de la biología molecular en América Latina ha estado marcado por contribuciones de muy alta calidad científica, aunque limitado 
a pocos países $\mathrm{y}$, dentro de éstos, a pocas instituciones de investigación científica; sin embargo, a pesar de este panorama, se puede esperar que la biología molecular, la ingeniería genética y las ciencias genómicas tengan un futuro brillante en los planes de desarrollo científico de los países de América Latina.

\section{Referencias}

Barrios, A. (1992) Eduardo De Robertis: un esbozo biográfico. SEDICCONICET, Argentina.

Birnbaumer, L. (2011) Retrospective: Héctor Norberto Torres (1935 - 2011). The American Society for Biochemistry and Molecular Biology. USA. (2011). http://www.asbmb.org/ asbmbtoday/201105/Retrospective/Torres/

Bonalume-Neto, R. (2010). Brazil boosts bioscience. Nature Biotechnology, 28, 191. DOI:10.1038/nbt310-191b.

Calva, E. (2009). El nacimiento de la Biología Molecular. Enciclopedia de las ciencias y la tecnología en México. http://www.izt.uam. $\mathrm{mx} / \operatorname{cosmosecm}$ /BIOLOGIA_MOLECULAR.html. (Acceso: 3 de mayo 2016).

Castañeda, G. (2002). Consideraciones sobre la historia de la bioquímica en México. Anales Médicos, 47(4), 232-239.

Centro de Ciencias Genómicas (2012). Nuestra Historia. http://www. ccg.unam.mx. (Acceso: 8 de mayo 2016).

Cereijido, M. (2000). La nuca de Houssay: la ciencia argentina entre Billiken y el exilio. Fondo de Cultura Económica, México.

De Block, M., Herrera-Estrella, L., Van Montagu, M., Schell, J. \& Zambryski, P. (1984). Expression of foreign genes in regenerated plants and in their progeny. EMBO Journal, 3(8), 1681-1689.

EMBL (2014). Argentina joins EMBL as associate member state. Press release. https://www.embl.de/aboutus/communication outreach/media_relations/2014/140422_BuenosAires/

Enciclopedia de Ciencias y Tecnologías en Argentina. https://cyt-ar. com.ar/cyt-ar/index.php/M\%C3\%A9todo_de_Galli_Mainini_ de_diagn $\% \mathrm{C} 3 \% \mathrm{~B} 3$ stico_precoz_del_embarazo (Acceso: 3 de junio 2016).

Farías, M., Revale, S., Mancini, E., Ordóñez, O., Turjanski, A. Cortez, N. \& Vázquez, M.P. (2011). Genome Sequence of Sphingomonas $s p$. S17, Isolated from an Alkaline, Hyperarsenic, and Hypersaline Volcano - Associated Lake at High Altitude in the Argentinian Puna. Journal of Bacteriology, 193(14), 36863687. DOI:1128/JB.05225 - 11.

Fernández, J., Mena, G. \& Santiesteban-Bonaechea, L. (2010) Tratamiento y recuperación del pie diabético grado 5 en la clasificación de Wagner tras aplicar el Herbertprot-P. Biotecnología aplicada, 27, 110 - 112.

Floras, N., Xiao, J., Berry, A., Bolivar, F. \& Valle, F. (1996). Pathway engineering for the production of aromatic compounds in Escherichia coli. Nature Biotechnology, 14(5), 620-623. DOI:10.1038/nbt0596-620

García, J. (2010). Siete maravillas de la Biotecnología cubana. CUBA LA GRAN NACIÓN. https://cubalagrannacion.wordpress. com/2010/06/13/siete-maravillas-de-la-biotecnologiacubana/. (Acceso 3 de mayo 2016).
González, V., Santamaría, R., Bustos, P., Hernández-González, I., Medrano-Soto, A. Moreno-Hagelsieb, G., Janga, S. C., Ramírez, M.A., Jiménez-Jacinto, V. Collado-Vides, J. \& Dávila, G. (2006). The partitioned Rhizobium etli genome: genetic and metabolic redundancy in seven interacting replicons. Proceedings of the National Academy of Sciences of USA, 103(10), 3834-3839. DOI:10.1073/pnas.0508502103

Herrera-Estrella, L., Depicker, A., Van Montagu, M. \& Schell, J. (1983) Expression of chimaeric genes transferred into plant cells using a Ti-plasmid-derived vector. Nature, 303(5914), 209-213. DOI: $10.1038 / 303209 a 0$

Hess, E. L. (1970). Origins of Molecular Biology. Science, 168, 664-669. IBYME - Instituto de Biología y Medicina Experimental, Argentina. http://www.ibyme.org.ar/. (Acceso: 4 de mayo 2016).

Instituto de Biotecnología (2017). http://www.ibt.unam.mx/. (Acceso: 4 de mayo 2017).

Instituto de Fisiología Celular UNAM (2017). http://www.ifc.unam. $\mathrm{mx} /$. (Acceso: 5 de mayo 2017).

Judson, H.F.(1979). The eighth day of creation: makers of the revolution in biology. Simon and Schuster, New York.

Kurth, D., Belfiore, C., Gorriti, M., Cortez, N., Farias, M. \& Albarracín V. H. (2015). Genomic and proteomic evidences unravel the UV-resistome of the poly-extremophile Acinetobacter sp. Ver3. Frontiers in Microbiology 2015, 6(328), 1-18. DOI: 10.3389/ fmicb. 2015.00328

Leloir Institute Foundation | Science Argentina. http://www.leloir.org. ar/en/. (Acceso: 3 mayo 2016).

Mantel, N., Girerd, Y., Geny, C., Bernard, I., Pontvianne, J., Lang, J. \& Barban, V. (2011). Genetic stability of a dengue vaccine based on chimeric yellow fever/dengue viruses. Vaccine, 29(38). 6629 - 6635. DOI: 10.1016/j.vaccine.2011.06.101. Epub 2011 Jul 13.

Memoria UNAM (2002). Dirección General de Planeación. UNAM. http://www.planeacion.unam.mx/Memoria/2002/. (Acceso: 4 de mayo 2016).

Monteiro-Vitorello, C.B., Camargo, L.E.A., Van Sluys, M.A., Kitajima, J.P., Truffi, D., do Amaral, A.M., Harakava, R., de Oliveira, J.C.F., Wood, D., de Oliveira, M.C., Miyaki, C., Takita, M.A., da Silva, A.C.R., Furlan, L.R., Carraro, D.M., Camarotte, G., Almeida, N.F., Carrer, H., Coutinho, L.L., El-Dorry, H.A., Ferro, M.I.T., Gagliardi, P.R., Giglioti, E., Goldman, M.H.S., Goldman, G.H., Kimura, E.T., Ferro, E.S., Kuramae, E.E., Lemos, E.G.M., Lemos, M.V.F., Mauro, S.M.Z., Machado, M.A., Marino, C.L., Menck, C.F., Nunes, L.R., Oliveira, R.C., Pereira, G.G., Siqueira, W., de Souza, A.A., Tsai, S.M., Zanca, A.S., Simpson, A.J.G., Brumbley, S.M. \& Setúbal, J.C. (2004). The genome sequence of the gram-positive sugarcane pathogen Leifsonia xyli subsp. xyli. Molecular Plant-Microbe Interactions, 17(8), 827- 836. DOI:10.1094/ MPMI.2004.17.8.827

Nemirovsky, S., Córdoba, M., Zaiat, J., Completa, S., Vega, P., González-Morón, D., Medina, N.M., Fabbro, M., Romero, S., Brun, B., Revale, S., Ogara, M.F., Pecci, A., Marti,M., Vazquez, M., Turjanski, A. \& Kauffman, M. A. (2015). Whole 
Genome Sequencing Reveals a De Novo SHANK3 Mutation in Familial Autism Spectrum Disorder. PLoS One 2015 Feb. 3, 10(2), e0116358. DOI: 10.1371/journal.pone.0116358. eCollection 2015.

Neuberger, M. (2002). "César Milstein(1927-2002)" Current Biology, 12(9), R308 - R310. DOI: http://dx.doi.org/10.1016/S09609822(02)00823-0

Nobel Lectures "Bernardo Houssay - Biographical". Physiology or Medicine 1942-1962, Elsevier Publishing Company, Amsterdam, 1964. http://www.nobelprize.org/nobel_prizes/ medicine/laureates/1947/houssay-bio.html (Acceso: 3 de mayo 2016).

Perkel, J. (2016). NIH dengue vaccine leaps into phase 3 studies. Nature Biotechnology, 34(5), 449. DOI: 10.1038/nbt0516-449

Quintero, R. (2007). Situación de la biotecnología y la genómica en México: investigación, formación de recursos humanos e industria. En: Educación, ciencia, tecnología y competitividad (Calva, J.L. coord.) 214-230. Porrúa - UNAM, México.

Rabinowicz, P. (2001). Genomics in Latin America: reaching the frontiers. Genome Research, 11, 319 - 322. DOI: 10.1101/ gr.179501

Ramírez, J.L., Gonzales, A., Cantú, J.M., Chávez-Crooker, P., Leya, J.C., Blamey, J.M., Cortés, H. \& Holmes, D. (2002). Latin American genome initiative: The creation of a network and web-based resource to aid and nurture genome biology in developing countries. Electronic Journal of Biotechnology, 5(3), 203 - 204. DOI: 10.4067/S0717-34582002000300002

Schnable, P.S., Ware, D., Fulton, R.S., Stein, J.C., Wei, F., Pasternak, S., Liang, C., Zhang, J., Fulton, L., Graves, T.A., Minx, P., Reily, A.D., Courtney, L., Kruchowski, S.S., Tomlinson, C., Strong, C., Delehaunty, K., Fronick, C., Courtney, B., Rock, S.M., Belter, E., Du, F., Kim, K., Abbott, R.M., Cotton, M., Levy, A., Marchetto, P., Ochoa, K., Jackson, S.M., Gillam, B., Chen, W., Yan, L., Higginbotham, J., Cardenas, M., Waligorski, J., Applebaum, E., Phelps, L., Falcone, J., Kanchi, K., Thane, T., Scimone, A., Thane, N., Henke, J., Wang, T., Ruppert, J., Shah, N., Rotter, K., Hodges, J., Ingenthron, E., Cordes, M., Kohlberg, S., Sgro, J., Delgado, B., Mead, K., Chinwalla, A., Leonard, S., Crouse, K., Collura, K., Kudrna, D., Currie, J., He, R., Angelova, A., Rajasekar, S., Mueller, T., Lomeli, R., Scara, G., Ko, A., Delaney, K., Wissotski, M., Lopez, G., Campos, D., Braidotti, M., Ashley, E., Golser, W., Kim, H., Lee, S., Lin, J., Dujmic, Z., Kim, W., Talag, J., Zuccolo, A., Fan, C., Sebastian, A., Kramer, M., Spiegel, L., Nascimento, L., Zutavern, T., Miller, B., Ambroise, C., Muller, S., Spooner, W., Narechania, A., Ren, L., Wei, S., Kumari, S., Faga, B., Levy, M.J., McMahan, L., Buren, P.V., Vaughn, M.W., Ying, K., Yeh, C.-T., Emrich, S.J., Jia, Y., Kalyanaraman, A., Hsia, A.-P., Barbazuk, W.B., Baucom, R.S., Brutnell, T.P., Carpita, N.C., Chaparro, C., Chia, J.-M., Deragon, J.-M., Estill, J.C., Fu, Y., Jeddeloh, J.A., Han, Y., Lee, H., Li, P., Lisch, D.R., Liu, S., Liu, Z., Nagel, D.H., McCann, M.C., SanMiguel, P., Myers, A.M., Nettleton, D., Nguyen, J., Penning, B.W., Ponnala, L., Schneider, K.L., Schwartz, D.C., Sharma, A., Soderlund, C.,
Springer, N.M., Sun, Q., Wang, H., Waterman, M., Westerman, R., Wolfgruber, T.K., Yang, L., Yu, Y., Zhang, L., Zhou, S., Zhu, Q., Bennetzen, J.L., Dawe, R.K., Jiang, J., Jiang, N., Presting, G.G., Wessler, S.R., Aluru, S., Martienssen, R.A., Clifton, S.W., McCombie, W.R., Wing, R.A. \& Wilson, R.K. (2009). The B73 maize genome: complexity, diversity, and dynamics. Science, 326, 1112-1115. DOI: 10.1126/science.1178534

Silva-Zolezzi, I., Hidalgo-Miranda, A., Estrada-Gil, J., FernandezLopez, J.C., Uribe-Figueroa, L., Contreras, A., Balam-Ortiz, E., Bosque-Plata, L. del, Velazquez-Fernandez, D., Lara, C., Goya, R., Hernandez-Lemus, E., Davila, C., Barrientos, E., March, S. \& Jimenez-Sanchez, G. (2009). Analysis of genomic diversity in Mexican Mestizo populations to develop genomic medicine in Mexico. Proceedings of the National Academy of Sciences of USA, 106(21), 8611-8616. DOI: 10.1073/pnas.0903045106 Simpson, A.J., Reinach, F.C., Arruda, P., Abreu, F.A., Acencio, M., Alvarenga, R., Alves, L.M., Araya, J.E., Baia, G.S., Baptista, C.S., Barros, M.H., Bonaccorsi, E.D., Bordin, S., Bové, J.M., Briones, M.R., Bueno, M.R., Camargo, A.A., Camargo, L.E., Carraro, D.M., Carrer, H., Colauto, N.B., Colombo, C., Costa, F.F., Costa, M.C., Costa-Neto, C.M., Coutinho, L.L., Cristofani, M., Dias-Neto, E., Docena, C., El-Dorry, H., Facincani, A.P., Ferreira, A.J., Ferreira, V.C., Ferro, J.A., Fraga, J.S., França, S.C., Franco, M.C., Frohme, M., Furlan, L.R., Garnier, M., Goldman, G.H., Goldman, M.H., Gomes, S.L., Gruber, A., Ho, P.L., Hoheisel, J.D., Junqueira, M.L., Kemper, E.L., Kitajima, J.P., Krieger, J.E., Kuramae, E.E., Laigret, F., Lambais, M.R., Leite, L.C., Lemos, E.G., Lemos, M.V., Lopes, S.A., Lopes, C.R., Machado, J.A., Machado, M.A., Madeira, A.M., Madeira, H.M., Marino, C.L., Marques, M.V., Martins, E.A., Martins, E.M., Matsukuma, A.Y., Menck, C.F., Miracca, E.C., Miyaki, C.Y., Monteriro-Vitorello, C.B., Moon, D.H., Nagai, M.A., Nascimento, A.L., Netto, L.E., Nhani, A., Nobrega, F.G., Nunes, L.R., Oliveira, M.A., de Oliveira, M.C., de Oliveira, R.C., Palmieri, D.A., Paris, A., Peixoto, B.R., Pereira, G.A., Pereira, H.A., Pesquero, J.B., Quaggio, R.B., Roberto, P.G., Rodrigues, V., de M Rosa, A.J., de Rosa, V.E., de Sá, R.G., Santelli, R.V., Sawasaki, H.E., da Silva, A.C., da Silva, A.M., da Silva, F.R., da Silva, W.A., da Silveira, J.F., Silvestri, M.L., Siqueira, W.J., de Souza, A.A., de Souza, A.P., Terenzi, M.F., Truffi, D., Tsai, S.M., Tsuhako, M.H., Vallada, H., Van Sluys, M.A., Verjovski-Almeida, S., Vettore, A.L., Zago, M.A., Zatz, M., Meidanis, J. \& Setubal, J.C. (2000). The genome sequence of the plant pathogen Xylella fastidiosa. The Xylella fastidiosa Consortium of the Organization for Nucleotide Sequencing and Analysis. Nature, 406(6792),151-159. DOI:10.1038/35018003

Soberón, X. (1997). Breve Semblanza Curricular de Francisco Bolívar Zapata. En: Francisco Bolivar Zapata. Obra Cientifica, 1, XXXIII-XV. El Colegio Nacional, México.

Stoppani, A. \& Milstein, C. (1957). "Essential role of thiol groups in aldehyde dehydrogenases". The Biochemical Journal, 67(3), 406-416. DOI: 10.1042/bj0670406

Tapia, R. Breve historia del Instituto de Fisiología Celular (http://www. ifc.unam.mx/pdfs/historia_ifc.pdf).(Acceso: 6 de mayo 2016) 
Traglia, G., Vilacoba, E., Almuzara, M., Diana, L., Iriarte, A., Centrón, D. \& Ramírez. M. (2014). Draft Genome Sequence of an Extensively Drug-Resistant Acinetobacter baumannii IndigoPigmented Strain. Genome Announcements, 2(6), e01146-14. DOI:10.1128/genomeA.01146-14

Vielle-Calzada, J.-P., Vega, O.M. de la, Hernández-Guzmán, G., Ibarra-Laclette, E., Alvarez-Mejía, C., Vega-Arreguín, J.C., Jiménez-Moraila, B., Fernández-Cortés, A., Corona-Armenta, G., Herrera-Estrella, L. \& Herrera-Estrella, A. (2009). The Palomero Genome Suggests Metal Effects on Domestication. Science, 326(5956), 1078. DOI:10.1126/science. 1178437
Villar, L., Dayan, G.H., Arredondo-García, J.L., Rivera, D.M., Cunha, R., Deseda, C., Reynales, H., Costa, M.S., MoralesRamírez, J.O., Carrasquilla, G., Rey, L.C., Dietze, R., Luz, K., Rivas, E., Miranda Montoya, M.C., Cortés Supelano, M., Zambrano, B., Langevin, E., Boaz, M., Tornieporth, N., Saville, M. \& Noriega, F. (2015). Efficacy of a Tetravalent Dengue Vaccine in Children in Latin America. The New England Journal of Medicine, 372, 113-123. DOI: 10.1056/ NEJMoa1411037

Wells, W. (2005). The discovery of synaptic vesicles. Journal of Cellular Biology, 168(1), 12-13. DOI: $10.1083 /$ jcb1681fta2 